\title{
DETECTION OF A LOW-ECCENTRICITY AND SUPER-MASSIVE PLANET TO THE SUBGIANT HD 38801*,
}

\author{
Hiroki Harakawa ${ }^{1}$, Bun'ei Sato ${ }^{2}$, Debra A. Fischer ${ }^{3,4}$, Shigeru Ida ${ }^{1}$, Masashi Omiya ${ }^{5}$, John A. Johnson ${ }^{6}$, \\ GEOFFrey W. MARCY ${ }^{7}$, Eri TOYOTA ${ }^{8}$, YASUNORI Hori ${ }^{1}$, AND ANDREW W. HOWARD ${ }^{7}$ \\ ${ }^{1}$ Department of Earth and Planetary Sciences, Tokyo Institute of Technology, 2-12-1 Ookayama, Meguro-ku, Tokyo 152-8551, Japan; harakawa@geo.titech.ac.jp \\ ${ }^{2}$ Global Edge Institute, Tokyo Institute of Technology, 2-12-1-S6-6 Ookayama, Meguro-ku, Tokyo 152-8550, Japan \\ ${ }^{3}$ Department of Physics \& Astronomy, San Francisco State University, San Francisco, CA 94132, USA \\ ${ }^{4}$ Department of Astronomy, Yale University, New Haven, CT 06511, USA \\ ${ }^{5}$ Department of Physics, Tokai University, 1117 Kitakaname, Hiratsuka, Kanagawa 259-1292, Japan \\ ${ }^{6}$ Institute for Astronomy, University of Hawaii, Honolulu, HI 96822, USA \\ ${ }^{7}$ Department of Astronomy, University of California, Berkeley, CA 94720, USA \\ ${ }^{8}$ Kobe Science Museum, 7-7-6 Minatojima-Nakamachi, Chuo-ku, Kobe, Hyogo 650-0046, Japan \\ Received 2009 September 12; accepted 2010 April 10; published 2010 April 29
}

\begin{abstract}
We report the detection of a large mass planet orbiting around the K0 metal-rich subgiant HD38801 $(V=8.26)$ by precise radial velocity (RV) measurements from the Subaru Telescope and the Keck Telescope. The star has a mass of $1.36 M_{\odot}$ and a metallicity of $[\mathrm{Fe} / \mathrm{H}]=+0.26$. The RV variations are consistent with a circular orbit with a period of 696.0 days and a velocity semiamplitude of $200.0 \mathrm{~m} \mathrm{~s}^{-1}$, which yield a minimum mass for the companion of 10.7 $M_{\text {JUP }}$ and a semimajor axis of $1.71 \mathrm{AU}$. Such super-massive objects with very low eccentricities and periods of hundreds of days are uncommon among the ensemble of known exoplanets.
\end{abstract}

Key words: planetary systems - stars: individual (HD 38801) - techniques: radial velocities - techniques: spectroscopic

\section{INTRODUCTION}

Since 1995, precise radial velocity (RV) measurements have unveiled more than 400 extrasolar planets from surveys that include roughly 3000 nearby stars (e.g., Udry \& Santos 2007). The planets show a great diversity in their physical attributes: masses of the planetary companions range from $4 M_{\oplus}$ $\left(\sim 0.013 M_{\mathrm{JUP}}\right)$ to larger than $13 M_{\mathrm{JUP}}$, with semimajor axes of 0.02-6 AU and orbital eccentricities of 0-0.9. A large number of planets have enabled us to discuss correlations between each orbital element and their masses in terms of planet formation and orbital evolution. For example, the diagram of orbital eccentricity against semimajor axis shows that eccentricities of planets with semimajor axes larger than $0.1 \mathrm{AU}$ are almost uniformly distributed from 0 to 1 , suggesting orbital evolution due to gravitational interaction between planets, while those inside $0.1 \mathrm{AU}$ tend to be damped below 0.1 due to tidal circularization. The diagram of eccentricity against planet mass also shows another possible hint for planet formation that supermassive planets ( $>5 M_{\mathrm{JUP}}$ ) tend to have relatively high eccentricity $(>0.3)$, while less massive planets have a wide range of eccentricities.

As the number of planet surveys has enlarged, diversity of planet-host stars has also been explored. Several surveys have focused on specified stellar properties, particularly stellar mass (Bonfils et al. 2005; Apps et al. 2010; Sato et al. 2008; Johnson et al. 2007) and metallicity (Fischer et al. 2005; Sozzetti et al. 2009), in order to investigate correlations between the stellar properties and planetary parameters such as stellar mass and occurrence rate of planets. As a result from these surveys, the statistical correlations between stellar properties and planet

\footnotetext{
* Based on data collected at the Subaru Telescope, which is operated by the National Astronomical Observatory of Japan.

$\dagger$ Based on observations obtained at the W. M. Keck Observatory, which is operated by the University of California and the California Institute of Technology. Keck time has been granted by NOAO and NASA.
}

parameters have begun to be unveiled. For example, occurrence rate of Jovian planets increases with stellar metallicity from $3 \%$ for stars with $[\mathrm{Fe} / \mathrm{H}]<0$ to $25 \%$ for those with $[\mathrm{Fe} / \mathrm{H}]>+0.3$ (Fischer et al. 2005), and also increases with stellar mass from $2 \%$ around $\mathrm{M}$ dwarfs $\left(<0.6 M_{\odot}\right)$ to approximately $9 \%$ around F and A stars (1.2-1.9 $M_{\odot}$; Johnson et al. 2007). Planetary mass could also be positively correlated with stellar metallicity and mass (Lovis \& Mayor 2007). Impacts of stellar properties on characteristics of planets, such as orbital eccentricity and multiplicity, are also expected to emerge as the number of planets grows.

The N2K program is a large-scale international exoplanetsearch project started in 2004, which consists of a sample of 2000 metal-rich solar-type stars (Fischer \& Valenti 2005). The search originally targeted short-period planets with a high-cadence observational strategy. However, it has also detected intermediate-period (18-3810 days) ones thanks to the long-term observations. From the collective N2K surveys, we have discovered 22 planets so far, which have a wide variety of masses $\left(0.22-13.1 M_{\mathrm{JUP}}\right)$ and orbital parameters $(a=$ 0.04-4.9 AU, $e=0-0.7$ ), and orbit around a variety of host stars $\left(-0.11<[\mathrm{Fe} / \mathrm{H}]<0.37,0.88<M / M_{\odot}<1.31\right.$; Wright et al. 2007; Johnson et al. 2006; Peek et al. 2009). The planets discovered from our surveys can thus contribute to our general understanding of planet formation and evolution depending on stellar properties.

Here we report the detection of an exoplanet orbiting the metal-rich solar-type subgiant HD38801 discovered from the N2K sample at the Subaru Telescope and Keck Observatory. The planet has a minimum mass of $10 M_{\mathrm{JUP}}$ and a low eccentricity. Such a planet has rarely been discovered so far around solar-type dwarfs and subgiants. We describe the characteristics of HD38801 in Section 2. In Section 3, we present our observations and the orbit of HD38801b. We summarize our results in Section 4 and discuss formation scenarios of such a low-eccentricity super-massive planet. 


\section{STELLAR CHARACTERISTICS}

HD38801 (HIP 27384) is listed in the Hipparcos catalogue (ESA 1997) as a K0IV star with a visual magnitude of $V=8.26$, and a color index $B-V=0.873$. The Hipparcos parallax of 10.06 mas corresponds to a distance of 99.4 pc which yields the absolute visual magnitude of $M_{V}=3.27$. A high-resolution spectroscopic analysis described in Valenti \& Fischer (2005) derived an effective temperature $T_{\text {eff }}=5222 \pm 44 \mathrm{~K}$, a surface gravity $\log g=3.84 \pm 0.1$, a rotational velocity $v \sin i=$ $0.54 \pm 0.5 \mathrm{~km} \mathrm{~s}^{-1}$, and a metallicity $[\mathrm{Fe} / \mathrm{H}]=0.26 \pm 0.03 \mathrm{dex}$ for the star. The bolometric luminosity of $L_{\text {star }}=4.56 \pm$ $0.47 L_{\odot}$ was derived from the $M_{V}$ and a bolometric correction of -0.195 (Flower 1996). From the bolometric luminosity, parallax, and $T_{\text {eff }}$, we derived a stellar radius of $2.53 \pm 0.13 R_{\odot}$ based on the Stefan-Boltzmann relation.

In order to estimate a stellar mass, we interpolated the metallicity, effective temperature, and luminosity onto the stellar interior model grids (Girardi et al. 2002). We adopted the threedimensional interpolation method described by Johnson et al. (2007) and applied 7\% uncertainty based on a comparison among different stellar model grids. Using the Spectroscopy Made Easy-derived stellar parameters, we estimated a mass of $1.36 \pm 0.09 M_{\odot}$ and an age of $4.67 \pm 2.56 \mathrm{Gyr}$ for HD38801. We measured $S_{H K}$ of -0.174 , which is core emission in the Ca II $H K$ lines relative to the continuum, to obtain chromospheric activity. We derived $\log R_{H K}^{\prime}=-5.0$, the ratio of flux from $S_{H K}$ to the bolometric stellar flux, which indicates that the star is chromospherically inactive. However, it is known that subgiants show "jitter" of 4-6 $\mathrm{m} \mathrm{s}^{-1}$, which is velocity scatter in excess of internal errors due to astrophysical sources such as pulsation and rotational modulation of surface features, even with such a low value of $\log R_{H K}^{\prime}$ (Johnson et al. 2007). We therefore adopt a jitter value of $6 \mathrm{~m} \mathrm{~s}^{-1}$ which is added in quadrature $\left(\sigma_{\mathrm{error}}^{2}=\sigma_{\mathrm{obs}}^{2}+\sigma_{\mathrm{jitter}}^{2}\right)$ to the formal velocity uncertainties for HD38801 when we fit a Keplerian orbit to the RV data. We summarize the parameters of HD38801 in Table 1.

\section{DOPPLER OBSERVATIONS AND KEPLERIAN FIT}

The Subaru Telescope's component of the N2K survey uses the high dispersion spectrograph (HDS) on the $8.2 \mathrm{~m}$ Subaru Telescope (Noguchi et al. 2002). We adopted the setup of StdI2b, which simultaneously covers a wavelength region of 3500$6100 \AA$ by a mosaic of two CCDs. The slit width was set to $0^{\prime \prime} .8$ for the first 3 observations (2005) and 0.6 for the last 15 data, giving a reciprocal resolution $(\lambda / \Delta \lambda)$ of 45,000 and 55,000 , respectively. Using this setup, we can obtain a signalto-noise ratio of $\mathrm{S} / \mathrm{N} \sim 150$ pixel s$^{-1}$ at $5500 \AA$ with $110-300 \mathrm{~s}$ exposure for our typical targets, $V \sim 8.5$ stars. We used an iodine absorption cell to provide a fiducial wavelength reference for precise RV measurements (Kambe et al. 2002; Sato et al. 2002). Although the cell was originally installed just behind the entrance of the spectrograph, it was moved in front of it in 2006. We used an analysis code of Sato et al. (2002) for modeling iodine superposed spectra and deriving stellar RVs, which is based on the standard method developed by Butler et al. (1996) and Valenti et al. (1995). Using the code and above spectrograph setting, we can achieve a Doppler precision of $4-5 \mathrm{~m} \mathrm{~s}^{-1}$.

Between 2006 September and 2009 January, we obtained 10 RV observations from the Keck 10 m Telescope with the HIRES spectrograph (Vogt et al. 1994). An iodine cell was placed in the optical path to provide both the precise wavelength solution and the point-spread function (PSF) of spectrograph instruments

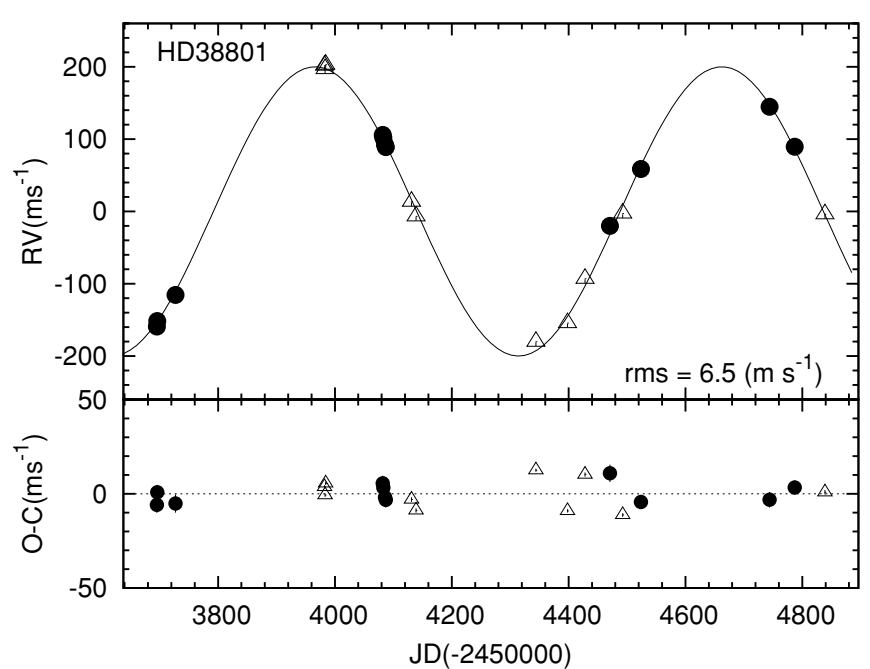

Figure 1. RVs for HD38801 derived from the Subaru Telescope (filled circles) and the Keck Telescope (opened triangles). With an orbital period of 696 days, a velocity semiamplitude of $200 \mathrm{~ms}^{-1}$, and a stellar mass of $1.36 M_{\odot}$, the indicating planet mass is $M \sin i=10.7 M_{J}$ and the semimajor axis is $1.7 \mathrm{AU}$. The eccentricity is fixed to 0 . The rms to the fit is $6.5 \mathrm{~m} \mathrm{~s}^{-1}$. The offsets of $-58.8 \mathrm{~m} \mathrm{~s}^{-1}$ and $20.5 \mathrm{~m} \mathrm{~s}^{-1}$ were applied to the Subaru RVs and the Keck RVs, respectively.

Table 1

Stellar Parameters for HD 38801

\begin{tabular}{lc}
\hline \hline \multicolumn{1}{c}{ Parameter } & Value (Error) \\
\hline$V$ & 8.26 \\
$M_{V}$ & 3.27 \\
$B-V$ & 0.873 \\
Spectral type & K0 IV(subgiant) \\
Distance $(\mathrm{pc})$ & 99.4 \\
$T_{\text {eff }}(\mathrm{K})$ & $5222(44)$ \\
$\log g$ & $3.84(0.1)$ \\
{$[\mathrm{Fe} / \mathrm{H}]$} & $0.26(0.03)$ \\
$v \sin i\left(\mathrm{~km} \mathrm{~s}{ }^{-1}\right)$ & $0.54(0.5)$ \\
$M_{*}\left(M_{\odot}\right)$ & $1.36(0.09)$ \\
$R_{*}\left(R_{\odot}\right)$ & $2.53(0.13)$ \\
$L_{*}\left(L_{\odot}\right)$ & $4.56(0.47)$ \\
Age $(\mathrm{Gyr})$ & $4.67(2.56)$ \\
$S_{H K}$ & -0.174 \\
$\log R_{H K}^{\prime}$ & -5.01 \\
\hline
\end{tabular}

(Marcy \& Butler 1992; Butler et al. 1996). We used the B5 decker corresponding to a slit width of 0 '.86 and a wavelength resolution of 65,000. Appropriate exposure times between 150 and $240 \mathrm{~s}$ were taken, and this results in an $\mathrm{S} / \mathrm{N}$ of 150 for respective observations. The typical precision of recent Keck observation is $1.5 \mathrm{~m} \mathrm{~s}^{-1}$.

We made the first observations for HD38801 at the Subaru Telescope and detected large RV variations. After that, the star has been monitored at the Subaru Telescope and the Keck Telescope for three years (from 2005 November to 2008 November) and a total of 20 data points were obtained. The observation dates, RVs, and instrumental uncertainties are listed in Table 2 and plotted in Figure 1.

To derive the best-fit Keplerian orbit to the RV data, we adopted the down-hill simplex algorithm (AMOEBA; Nelder $\&$ Mead 1965) to reduce $\chi^{2}$ which has multiple parameters (orbital period $P$, time of periastron passage $T_{c}$, eccentricity $e$, velocity semiamplitude $K_{1}$, and argument of periastron $\omega)$ and obtained the best-fit parameters of single Keplerian orbit. The stellar jitter of $6 \mathrm{~m} \mathrm{~s}^{-1}$ was added in quadrature to 


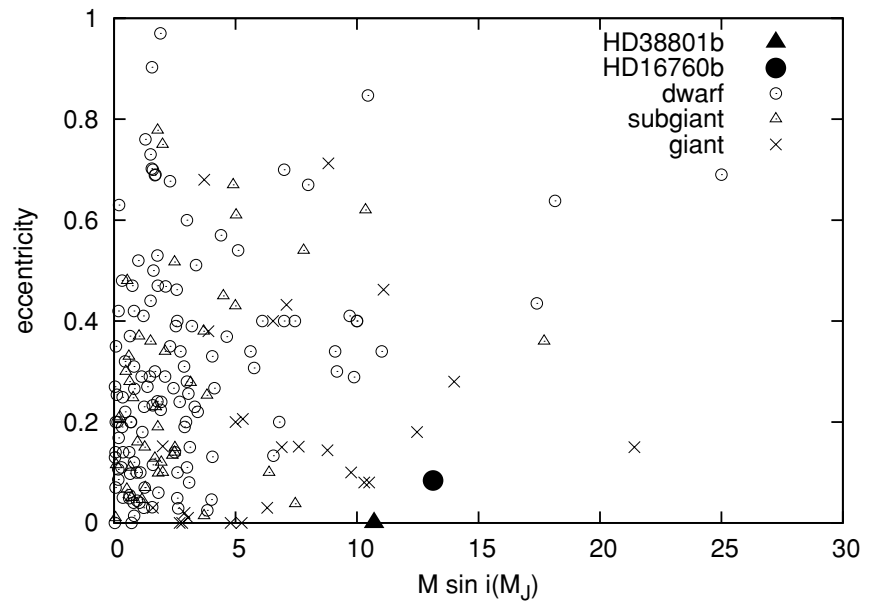

Figure 2. Correlation diagram of the eccentricity and the minimum mass of the planets with above 14 day orbit. HD38801b is plotted with a filled triangle. HD16760b (filled circle), main-sequence stars (opened circles), subgiants (opened triangles), and giants (crosses) are also plotted. The companion's properties of HD16760 are given in Sato et al. (2009)—http://exoplanet.eu.

Table 2

RVs for HD38801

\begin{tabular}{lrcc}
\hline \hline $\begin{array}{l}\text { Observation Date } \\
(\mathrm{JD}-2,450,000)\end{array}$ & \multicolumn{1}{c}{$\begin{array}{c}\mathrm{RV} \\
\left(\mathrm{m} \mathrm{s}^{-1}\right)\end{array}$} & $\begin{array}{c}\text { Uncertainties } \\
\left(\mathrm{m} \mathrm{s}^{-1}\right)\end{array}$ & Observatory \\
\hline 3695.0902 & -159.0 & 4.2 & Subaru \\
3695.9883 & -151.3 & 4.0 & Subaru \\
3727.0304 & -115.4 & 4.8 & Subaru \\
3982.1185 & 201.4 & 0.9 & Keck \\
3983.1122 & 196.8 & 0.9 & Keck \\
3984.1088 & 202.8 & 0.9 & Keck \\
4081.9436 & 105.9 & 4.0 & Subaru \\
4082.9788 & 101.9 & 4.7 & Subaru \\
4085.9284 & 92.2 & 4.8 & Subaru \\
4087.0406 & 89.0 & 4.1 & Subaru \\
4130.8895 & 13.4 & 1.2 & Keck \\
4138.9172 & -6.9 & 1.2 & Keck \\
4344.1185 & -180.3 & 0.8 & Keck \\
4398.0809 & -154.4 & 0.9 & Keck \\
4427.9607 & -93.3 & 1.1 & Keck \\
4470.9194 & -19.9 & 4.6 & Subaru \\
4492.7365 & -3.1 & 1.0 & Keck \\
4523.7729 & 59.0 & 3.5 & Subaru \\
4744.0407 & 145.0 & 4.1 & Subaru \\
4787.0288 & 89.6 & 3.9 & Subaru \\
4838.9756 & 4.0 & 1.2 & Keck \\
\hline
\end{tabular}

the measurement uncertainties. At first fit, when all of these parameters were let free, we obtained very low eccentricity of 0.04 and poorly constrained $\omega$. So we made another fitting with the fixed values of $e$ and $\omega$ set to 0 . The best-fit parameters thus obtained are listed in Table 3, and the Keplerian fit is overplotted on the RV data in Figure 1. We obtained a period of $P=696.3 \pm 2.7$ days and a velocity semiamplitude of $200.0 \pm 3.9 \mathrm{~m} \mathrm{~s}^{-1}$. The rms scatter to this fit is $6.5 \mathrm{~m} \mathrm{~s}^{-1}$ and $\left(\chi_{v}^{2}\right)^{1 / 2}=1.2$ as a reduced $\chi$. The uncertainties in the orbital parameters were estimated by a bootstrap Monte Carlo approach. The RV residuals from the best-fit Keplerian curve are scrambled and added back to the original measurements, and then re-fit and derive new parameters. Iterating 10,000 times, we obtained the $1 \sigma$ value of each parameter. With a stellar mass of $1.36 \pm 0.09 M_{\odot}$, we obtained a minimum mass for the
Table 3

Spectroscopic Orbital Solution for HD38801b

\begin{tabular}{lc}
\hline \hline \multicolumn{1}{c}{ Parameter } & Value (Error) \\
\hline$P($ days $)$ & $696.3(2.7)$ \\
$T_{c}(\mathrm{JD})$ & $2,453,966.0(2.1)$ \\
Eccentricity & 0 (fixed) \\
$\omega$ & 0 (fixed) \\
$K_{1}\left(\mathrm{~m} \mathrm{~s}^{-1}\right)$ & $200.0(3.9)$ \\
$a_{1} \sin i\left(10^{-3} \mathrm{AU}\right)$ & $13(0.27)$ \\
$a_{p}(\mathrm{AU})$ & $1.70(0.037)$ \\
$M \sin i\left(M_{\mathrm{JUP}}\right)$ & $10.7(0.5)$ \\
$N_{\text {obs }}$ & 21 \\
$\operatorname{rms}\left(\mathrm{m} \mathrm{s}^{-1}\right)$ & 6.5 \\
$\operatorname{Reduced}\left(\chi_{v}^{2}\right)^{1 / 2}$ & 1.2 \\
\hline
\end{tabular}

companion of $M \sin i=10.7 \pm 0.5 M_{\mathrm{JUP}}$ and a semimajor axis of $a_{p}=1.70 \pm 0.03 \mathrm{AU}$.

As seen in Figure 1, the residuals to the Keplerian fit in JD2454300-2454500 exhibit larger scatter than other parts. We performed a periodogram analysis (Scargle 1982) to the residuals. However, we found no significant peaks in the periodogram at this stage. More dense sampling of data will help verify the existence of any periodicity in the residuals and its origin.

\section{SUMMARY AND DISCUSSION}

We here reported the detection of a large-mass planet in an almost circular orbit around a high-metallicity K0 IV type star HD38801 from the precise RV observations at the Subaru Telescope and the Keck Telescope. The star has a mass of $1.36 M_{\odot}$ and a metallicity of $[\mathrm{Fe} / \mathrm{H}]=0.26$. The planet is a new sample around a metal-rich and relatively high-mass star, which plays an important role in investigating the dependence of planets on host star's properties.

One remarkable feature of this planet is its low orbital eccentricity $(e \sim 0)$ despite the large mass $\left(M \sin i=10.7 M_{\mathrm{JUP}}\right)$ and the intermediate orbital distance $(a=1.7 \mathrm{AU})$. Such a planet with low eccentricity $(e<0.1)$ and large mass $(M \sin i>$ $\left.10 M_{\mathrm{JUP}}\right)$ has been rarely discovered so far in the regions of $0.1 \mathrm{AU}<a<5 \mathrm{AU}$ around FGK dwarfs and subgiants. Only HD16760b $\left(M \sin i=13 M_{\mathrm{JUP}}, a=1.08 \mathrm{AU}, e=0.084\right)$, which was recently discovered from the Subaru Telescope and the Keck Telescope (Bouchy et al. 2009; Sato et al. 2009), falls into this category, besides HD38801b (Figure 2). In the coreaccretion model, massive companions are generally formed with circular orbits because of protoplanetary disk's circular motion. The circular orbit of HD38801b is consistent with this scenario. Tidal interaction between the host star and the planet cannot explain the circular orbit because the stellar radius is only $2.5 R_{\odot}$ ( $\sim 0.01 \mathrm{AU})$, which is too small to circularize the planetary orbit at 1.7 AU. Contrary to HD $38801 \mathrm{~b}$, most of the intermediateperiod ( $>18$ days) and large-mass ( $>5 M_{\mathrm{JUP}}$ ) planets discovered around FGK dwarfs and subgiants so far tend to reside in eccentric orbits. The origin of such planets still remains to be solved. It is generally thought that eccentric planets are formed by planet-planet scattering in multiple planetary systems. The scenario suggests formation of multi super-massive planets in a system and predicts the existence of scattered outer companions as massive as inner ones, which can be good targets for direct imaging. Other scenarios such as giant impact (Baraffe et al. 2008), disk instability (Boss 1998), and Kozai mechanism when they are in binary systems (Kozai 1962), have also 
been proposed to explain the formation of such massive eccentric planets. Interestingly, planets with similar properties to HD38801b have been discovered around intermediate-mass giants. Comparing populations of such planets between different types of stars would provide a hint on formation scenario and evolution for the planets.

The high metallicity and the evolutionary stage of subgiant for HD38801 are another interesting feature of this system. It is well known that the detection rate of giant planets shows positive correlation with the metallicity of the host stars (Fischer $\&$ Valenti 2005). Two scenarios have been proposed to explain the origin of the correlation; one is that more metal-rich stars tend to form more giant planets, and the other is that the stellar surface is polluted by planetary debris (e.g., Ida \& Lin 2004). At the early stage of the main sequence, a stellar surface could be polluted due to the accretion of planetesimals, and protoplanets from the debris disk and metallicity of the stellar surface could be enhanced. However, if the star has deep convective envelope, the metal-enhanced surface can be mixed with original stellar material and diluted by the convective flow. Thus, the existence of a giant planet around a metal-rich evolved star as the subgiant HD38801, which probably has deep convective envelope, favors the former scenario, that is, giant planets tend to form in metalrich environment (Fischer \& Valenti 2005). It should be noted, however, that many planets have been discovered even in metalpoor $([\mathrm{Fe} / \mathrm{H}]<0)$ giants, more evolved stars than subgiants, and no significant planet-metallicity correlation can be seen among them. Pasquini et al. (2007) proposed that the lack of metal-rich tendency is due to dilution by deeper convective envelope of giants than that of dwarfs and subgiants. To make sure the origin of the high metallicity of planet-harboring dwarfs, it is important to increase the number of planets discovered around evolved stars with $<1.5 M_{\odot}$, for which we can detect planets in all of the three evolutionary stages, dwarfs, subgiants, and giants, by the precise Doppler technique.

We extend our gratitude to Akito Tajitsu and Tae-Soo Pyo for their great expertise and support of HDS observations from the Subaru Telescope. We also gratefully acknowledge the efforts and dedication of all Keck Observatory staff. This research has made use of the Simbad database, operated at CDS, Strasbourg, France. We thank all Hawaiian people for their hospitality and native Hawaiian ancestry on whose sacred mountain of Mauna Kea we are privileged to be guests. Without their generous supports, we would not be able to publish our new planet.

\section{REFERENCES}

Apps, K., et al. 2010, PASP, 122, 156

Baraffe, I., Chabrier, G., \& Barman, T. 2008, A\&A, 482, 315

Bonfils, X., et al. 2005, A\&A, 443, L15

Boss, A. P. 1998, ApJ, 503, 923

Bouchy, F., et al. 2009, A\&A, 505, 853

Butler, R. P., Marcy, G. W., Williams, E., McCarthy, C., Dosanjh, P., \& Vogt, S. S. 1996, PASP, 108, 500

ESA 1997, The Hipparcos and Tycho Catalogues (ESA SP-1200; Noordwijk: ESA)

Fischer, D. A., \& Valenti, J. 2005, ApJ, 622, 1102

Fischer, D. A., et al. 2005, ApJ, 620, 481

Flower, P. J. 1996, ApJ, 469, 355

Girardi, L., Bertelli, G., Bressan, A., Chiosi, C., Groenewegen, M. A. T., Marigo, P., Salasnich, B., \& Weiss, A. 2002, A\&A, 391, 195

Ida, S., \& Lin, D. N. C. 2004, ApJ, 616, 567

Johnson, J. A., et al. 2006, ApJ, 647, 600

Johnson, J. A., et al. 2007, ApJ, 665, 785

Kambe, E., et al. 2002, PASJ, 54, 865

Kozai, Y. 1962, AJ, 67, 591

Lovis, C., \& Mayor, M. 2007, A\&A, 472, 657

Marcy, G. W., \& Butler, R. P. 1992, PASP, 104, 270

Nelder, J. A., \& Mead, R. 1965, Comput. J., 7, 308

Noguchi, K., et al. 2002, PASJ, 54, 855

Pasquini, L., Döllinger, M. P., Weiss, A., Girardi, L., Chavero, C., Hatzes, A. P., da Silva, L., \& Setiawan, J. 2007, A\&A, 473, 979

Peek, K. M. G., et al. 2009, PASP, 121, 613

Sato, B., Kambe, E., Takeda, Y., Izumiura, H., \& Ando, H. 2002, PASJ, 54, 873 Sato, B., et al. 2008, PASJ, 60, 1317

Sato, B., et al. 2009, ApJ, 703, 671

Scargle, J. D. 1982, ApJ, 263, 835

Sozzetti, A., Torres, G., Latham, D. W., Stefanik, R. P., Korzennik, S. G., Boss, A. P., Carney, B. W., \& Laird, J. B. 2009, ApJ, 697, 544

Udry, S., \& Santos, N. C. 2007, ARA\&A, 45, 397

Valenti, J. A., Butler, R. P., \& Marcy, G. W. 1995, PASP, 107, 966

Valenti, J. A., \& Fischer, D. A. 2005, VizieR Online Data Catalog, 215, 90141

Vogt, S. S., et al. 1994, Proc. SPIE, 2198, 362

Wright, J. T., et al. 2007, ApJ, 657, 533 